\title{
Estudo cinético de decomposição térmica do óleo de girassol suportado em material mesoporoso.
}

\author{
A. M. M. ARAÚJO ${ }^{1}$, A. S. ARAÚJO², V. J. FERNANDES JÚNIOR ${ }^{2}$ e L. D. SOUZA ${ }^{3}$. \\ ${ }^{1}$ Universidade Federal do Rio Grande do Norte, Programas de Pós Graduação em Ciências e \\ Engenharia do Petróleo. \\ ${ }^{2}$ Universidade Federal do Rio Grande do Norte, Departamento de Química. \\ ${ }^{3}$ Universidade do Estado do Rio Grande do Norte, Departamento de Química \\ E-mail para contato: aruzzamabel@gmail.com
}

\begin{abstract}
RESUMO - 0 material mesoporoso tipo Al-MCM-41 foi sintetizado hidrotermicamente e caracterizado pelos métodos de difração de raios-X, microscopia eletrônica de varredura, espectroscopia eletrônica com transformada de Fourrier e adsorção de nitrogênio. Ainda foi realizado o estudo do comportamento termogravimétrico do óleo de girassol sobre o catalisador mesoporoso. 0 material mesoporoso Al-MCM-41 apresentou formação hexagonal unidimensional. Com base nas curvas integrais das análises termogravimétricas e o método cinético de Vyazovkin, foram estimados a energia de ativação, a conversão e a degradação do óleo em função da temperatura. 0 estudo do comportamento cinético do óleo de girassol com o catalisador mostrou energia de ativação menor que à energia de ativação do óleo de girassol puro.
\end{abstract}

\section{INTRODUÇÃO}

O uso de biocombustíveis cresce com a demanda de alternativas por combustíveis limpos. Atualmente, as pesquisas estão voltadas a melhoria do processo de craqueamento e o uso de catalisadores é uma das alternativas estudadas. A ação desoxigenante dos catalisadores tem mostrado que as frações líquidas obtidas apresentam propriedades semelhantes à gasolina e ao diesel de petróleo. Do ponto de vista da catálise, estudos recentes apontam uma maior seletividade à fração semelhante ao diesel à medida que há um aumento no diâmetro de poros e, para catalisadores com um menor diâmetro de poros a seletividade é maior para a gasolina.

Como base para a realização do craqueamento em escala de bancada foi realizado o estudo cinético pelo método de Vyazovkin e Goryachko, que desenvolveram um método cinético (model-free kinetic) onde as taxas de aquecimento permitem avaliar múltiplas reações cinéticas simples e complexas ao mesmo tempo. 
Com o passar dos anos, as técnicas termoanalíticas adquiriram importância crescente em todas as áreas de conhecimento na química aplicada. Esse incremento na utilização dessa metodologia, realmente dotada de grande potencialidade foi favorecido pela disponibilidade de instrumentos controlados por microprocessadores, capazes de fornecer informações quanto ao comportamento térmico dos materiais de forma precisa e em um tempo relativamente curto.

A análise Térmica possibilita uma ampla faixa de aplicação para medidas de propriedades físicas, estudo de reações químicas, avaliação da estabilidade térmica, determinação da composição de materiais e desenvolvimento de metodologia analítica.

As técnicas termoanalíticas podem ser consideradas como um instrumento essencial nos estudos da cinética e mecanismo das substâncias. Para que uma técnica térmica possa ser considerada termoanalítica deve obedecer aos seguintes critérios:

- Medir uma propriedade física;

- Expressar a medida, direta ou indiretamente, em função da temperatura;

- Realizar a medida sob um controle de temperatura.

Os principais métodos termogravimétricos classificam-se em dinâmico, isotérmico e quase isotérmico. No método dinâmico, a perda de massa é registrada continuamente à medida que a temperatura aumenta. No método isotérmico, a temperatura medida é mantida constante, sendo registrada a variação da massa em função do tempo. Já no método quase isotérmico, a partir do momento em que começa incidir a perda de massa da amostra, a temperatura é medida constantemente, até que a massa se estabilize novamente. Como mostra a Figura 1 Dantas; Souza; Conceição, (2006).

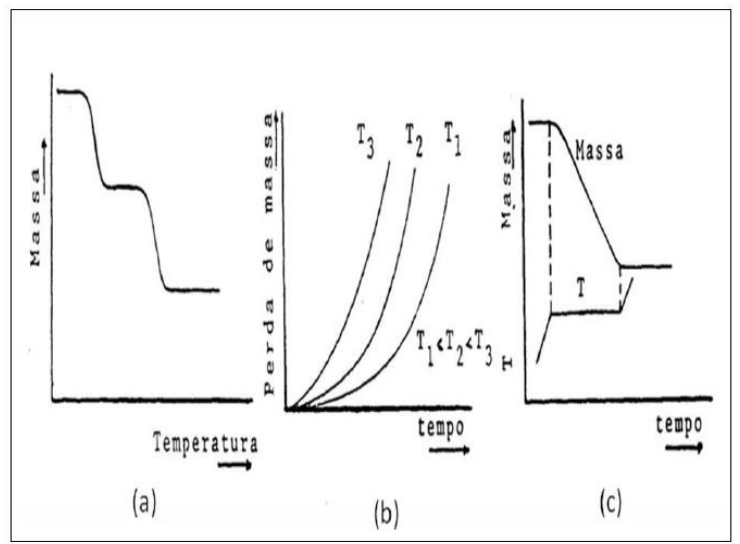

Figura 1 - Curvas típicas dos principais métodos termogravimétricos: a) dinâmico; b) isotérmico c) quase-isotérmico

Dantas; Souza; Conceição (2006) 
A variação da massa é realizada utilizando-se uma termobalança, que consiste na combinação de uma microbalança eletrônica adequada com um forno e um sistema de temperatura controlada, que permite a pesagem contínua de uma amostra em função da temperatura à medida que a amostra é termodinamicamente aquecida ou resfriada. As temperaturas do forno e da amostra são determinadas através de um termopar e o sensor deve estar localizado aproximadamente 1 a $2 \mathrm{~mm}$ próximo da amostra, de modo a minimizar os erros referentes a limitações difusionais na transferência de calor, nesse caso mais presentes devido a problemas associados com a convecção e condução de calor Gonzalez et al, (2008).

O porta-amostras é geralmente constituído de alumínio, alumina, platina, níquel, quartzo, tungstênio, grafite e cobre, deve ser escolhido de acordo com a amostra a ser analisada e com a temperatura máxima de aquecimento aplicada à amostra. A massa de amostra pode variar entre $10 \mathrm{mg}$ até $10 \mathrm{~g}$, dependendo do equipamento utilizado. A atmosfera que circunda a amostra pode ser controlada, possibilitando trabalhar com atmosfera estática ou dinâmica à pressão ambiente, sob pressão ou a vácuo. Os gases utilizados podem ser nitrogênio e argônio (inertes), oxigênio (oxidantes) ou corrosivos Dantas; Souza; Conceição, (2006).

A termogravimetria também permite usar sistemas simultâneos, dentre os quais se podem destacar: termogravimetria-cromatografia gasosa (TG-GC); termogravimetria-espectroscopia de massa (TG-MS); termogravimetria-cromatografia à gas-espectroscopia de massa (TG-GC-MS).

O presente trabalho relata a síntese e caracterização do Al-MCM-41 e um estudo cinético da sua aplicação no craqueamento do óleo de girassol/Al-MCM-41.

\section{MATERIAIS E MÉTODOS}

Síntese do Al-MCM-41 razão $\mathrm{Si} / \mathrm{Al}=50$

A peneira molecular mesoporosa Al-MCM-41 foi sintetizada pelo método hidrotérmico partindo-se de procedimentos experimentais adaptados de diversos trabalhos BECK et al, (1992) e ARAUJO et al, (2004) seguindo a composição molecular abaixo:

\section{CTMABr: 2 NaOH: $4 \mathrm{SiO}_{2}: 200 \mathrm{H}_{2} \mathrm{O}$.}

\section{Caracterizações físico-químicas do catalisador}

A amostra de Al-MCM-41 foi caracterizada por Difração de Raios-X (DRX), Adsorção do Nitrogênio e Análise Termogravimétrica segundo metodologia descrita na literatura, Melo, (2010). 


\section{Estudo cinético}

As análises termogravimétricas (TG/DTG) do catalisador foram realizadas em um equipamento Mettler Toledo, modelo 851, com razão de aquecimento de $10^{\circ} \mathrm{C} \cdot \mathrm{min}^{-1}$, utilizandose atmosfera de hélio, com vazão de $25 \mathrm{~mL} \cdot \mathrm{min}^{-1}$, cadinhos de alumina e aproximadamente 20 mg de amostra.

Realizou-se o estudo cinético para a degradação do óleo de girassol puro e do óleo de girassol com o catalisador AlMCM-41, sob a atmosfera do hélio com três diferentes razões de aquecimento $\left(5 ; 10\right.$ e $20^{\circ} \mathrm{C}$ min-1). Usando curvas integrais da $\mathrm{TG}$ e o método cinético de Vyazovkin, foram estimadas a energia de ativação, as taxas de conversão e o tempo degradação do óleo em função do tempo.

\section{RESULTADOS E DISCUSSÃO}

\section{Caracterização do material obtido}

Como se pode observar na Figura 1, o difratograma de Raios-X apresenta a característica típica do material com estrutura hexagonal unidimensional, do tipo Al-MCM-41 com razão Si/Al $=50$.

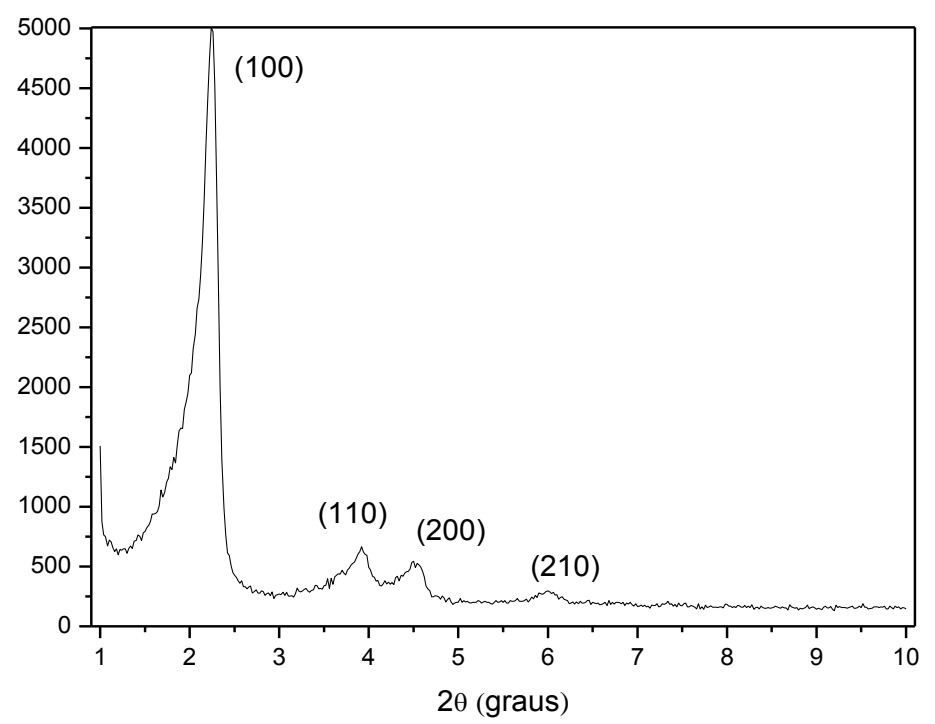

Figura 1. Difratograma de raios-X do material Al-MCM-41.

Foi observado, a presença de 4 picos característicos de difração do plano, cujos índices são (100), (110), (200) e (210). Segundo a literatura BECK, J.S, (1992) esses planos, são característicos de um sistema hexagonal bem estruturado, comum a materiais da família MCM41. 
A área superficial do material mesoporoso que foi utilizado na pirólise catalítica do óleo de girassol foi de $853,7 \mathrm{~m}^{2} / \mathrm{g}$. As propriedades texturais obtidas a partir dos resultados de adsorção do material utilizado são mostradas na Tabela 1.

Tabela 1. Propriedades texturais do catalisador Al-MCM-41 sintetizado

\begin{tabular}{cccccc}
\hline Amostra & $A_{0}(\mathrm{~nm})$ & $D_{p}(\mathrm{~nm})$ & $W_{t}(\mathrm{~nm})$ & $V_{p}\left(\mathrm{~cm}^{3} / \mathrm{g}\right)$ & $\mathrm{S}_{\mathrm{BET}}\left(\mathrm{m}^{2} / \mathrm{g}\right)$ \\
\hline AlMCM-41 & $\mathbf{4 , 7 9}$ & $\mathbf{1 , 5 4 0}$ & $\mathbf{3 , 2 5}$ & $\mathbf{0 , 1 1}$ & $\mathbf{8 5 3 , 7 0}$ \\
\hline
\end{tabular}

$\mathrm{A}_{0}=$ Parâmetro de rede; $\mathrm{Dp}=$ Diâmetro do poro; $\mathrm{Wt}=$ Espessura da parede de sílica; $\mathrm{Vp}=$ Volume de poros; $\mathrm{S}_{\mathrm{BET}}=$ área superficial através do método de BET.

\section{Estudo cinético}

As curvas TG do óleo de girassol e do óleo com catalisador (óleo/Al-MCM-41) são mostrados na Figura 2. A perda mássica, comum às duas curvas TG, ocorreu na faixa de $300-$ $496{ }^{\circ} \mathrm{C}$, atribuída à vaporização dos compostos (ácidos graxos) presentes nos dois óleos do óleo de girassol. No entanto, observa-se na Tabela 2 que a temperatura inicial das perdas varia, havendo uma diminuição ao ser adicionado o catalisador, sugerindo assim que os compostos presentes no óleo/Al-MCM-41 tem massa molecular menor que os do óleo, ou seja foram degradados, indicando a atividade catalítica deste sólido durante a pirólise do óleo de girassol. Esse comportamento pode ser explicado pela acidez do Al-MCM-41.

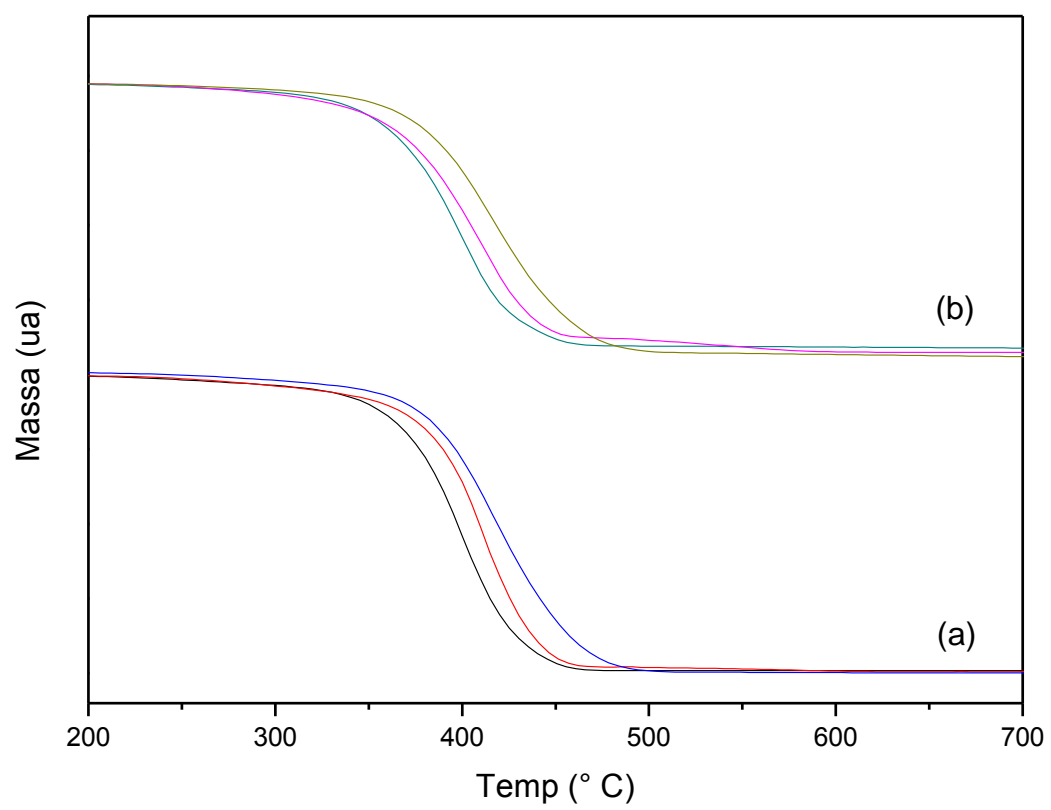

Figura 2 - (a) Curvas de TG do óleo de girassol, (b) óleo/AlMCM-41 com diferentes razões de aquecimento $\beta \mathrm{i}\left(\mathrm{i}=5 ; 10\right.$ e $20^{\circ} \mathrm{C}$ min- 1$)$. 
Como se pode observar na Tabela 2, as amostras apresentam uma única perda acentuada na faixa de temperatura de $300-496{ }^{\circ} \mathrm{C}$, de modo que este intervalo foi selecionado para estudos cinéticos.

Tabela 2 - Faixas de temperaturas da pirólise do óleo/AlMCM-41 e óleo de girassol de acordo com as curvas TG.

Amostras

Óleo de Girassol

Óleo/Al-MCM-41
Faixas de Temperatura $\left({ }^{\circ} \mathrm{C}\right)$

$340-496$

300-466

A Figura 3 mostra a conversão obtida em função da degradação do óleo em função da temperatura para o óleo puro e na presença de $20 \%$ em peso do catalisador. A degradação na presença do Al-MCM-41 foi mais eficiente, pois necessitou de menor energia.

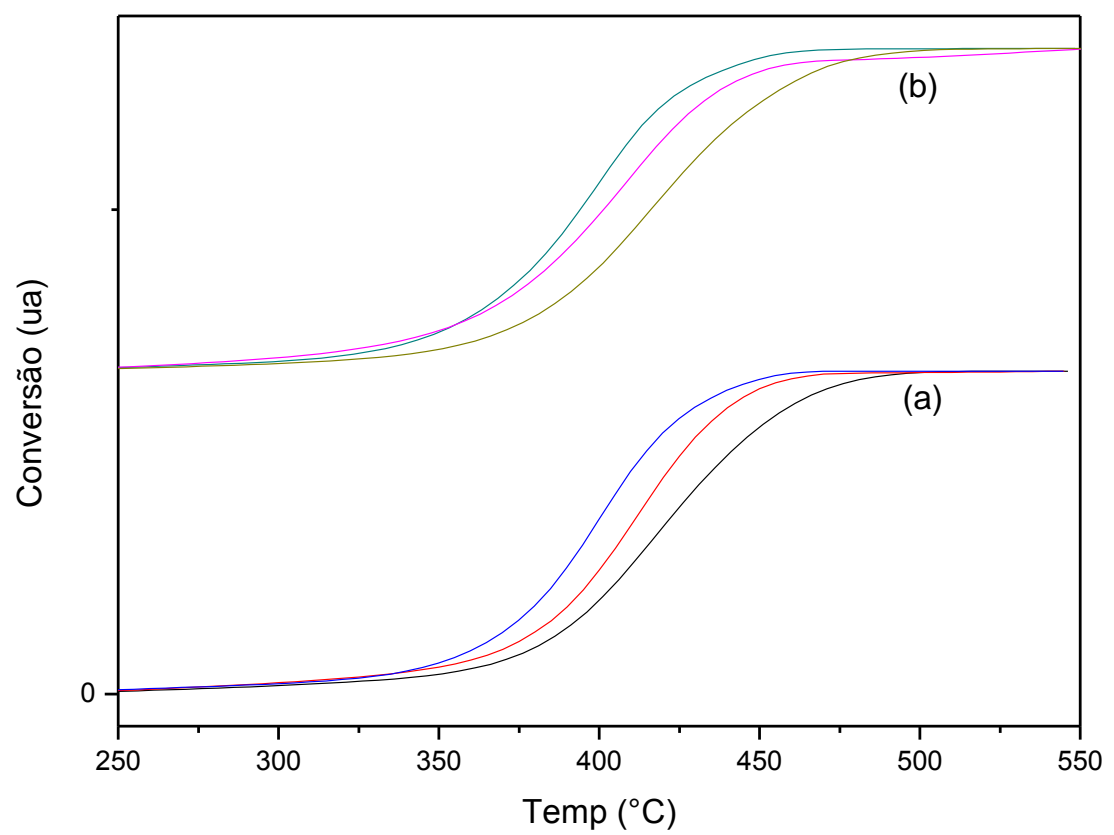

Figura 3 - (a)Conversão do óleo de girassol, (b) óleo/AlMCM-41 em função da temperatura com diferentes razões de aquecimento $\alpha \mathrm{i}\left(\mathrm{i}=5 ; 10\right.$ e $20^{\circ} \mathrm{C}$ min- 1$)$.

Vyazovkin e Goryachko (1992) desenvolveram um método cinético (model-free kinetic) onde as taxas de aquecimento permitem avaliar múltiplas reações cinéticas simples e complexas ao mesmo tempo. Sabe-se que a taxa de reação de uma reação térmica e catalítica depende de conversão $(\alpha)$, temperatura $(\mathrm{T})$ e tempo $(\mathrm{t})$. Para cada processo, a taxa de reação em função da conversão, $\mathrm{f}(\alpha)$, é diferente e deve ser determinada a partir dos dados experimentais. Para as reações individuais, a avaliação de $\mathrm{f}(\alpha)$, na ordem “n” é possível. Para reações complexas, tal 
como as que ocorrem na pirólise do óleo, por exemplo, a avaliação de $\mathrm{f}(\alpha)$ é complicada e, em geral desconhecida. Neste caso, para obter informações confiáveis e consistentes sobre a cinética global do processo, o método do model-free kinetics foi aplicado baseado na teoria Vyazovkin.

Esta teoria é baseada em uma técnica isoconversional computacional que calcula a energia de ativação efetiva (Ea) em função da conversão $(\alpha)$ de uma reação química, $\mathrm{E}=\mathrm{f}(\alpha)$. Os métodos que fazem uso do princípio isoconversional afirmam que em uma medida constante de conversão, a taxa de reação é uma função apenas da temperatura de forma que:

$$
\left[\frac{d \ln (d \alpha / d t)}{d T^{-1}}\right]=-\frac{E_{a}}{R}
$$

Na equação (1), Ea é o parâmetro de Arrhenius (energia de ativação aparente), $\mathrm{R}$ é a constante do gás, $\mathrm{T}$ é a temperatura, t é o tempo, e $\alpha$ é a medida de conversão, que pode ser determinada a partir da análise de TG como uma perda de massa fracionada. $\mathrm{O}$ model-free kinetics é um programa baseado na teoria Vyazovkin para estudos de decomposição da reação complexa. A abordagem segue todos os pontos de conversão de múltiplas experiências, em vez de um único. A reação química é medida pelo menos em três diferentes taxas de aquecimento ( $\beta$ ) e as respectivas curvas de conversão são calculadas fora das curvas TG medidas Vyazovkin \& Goryachko, (1992).

A energia de ativação para a decomposição térmica e termocatalítica do óleo de girassol esta mostrada na Figura 4. Para o óleo a energia de ativação a $45 \%$, foi de $249 \mathrm{Kj}_{\mathrm{mol}}{ }^{-1}$, enquanto que para a amostra óleo/Al-MCM-41 a energia de ativação foi menor, $221 \mathrm{Kj}_{\mathrm{mol}}{ }^{-1}$, baseado nessa diminuição, pode-se supor que os catalisadores mesoporosos são promissores na degradação do óleo vegetal.

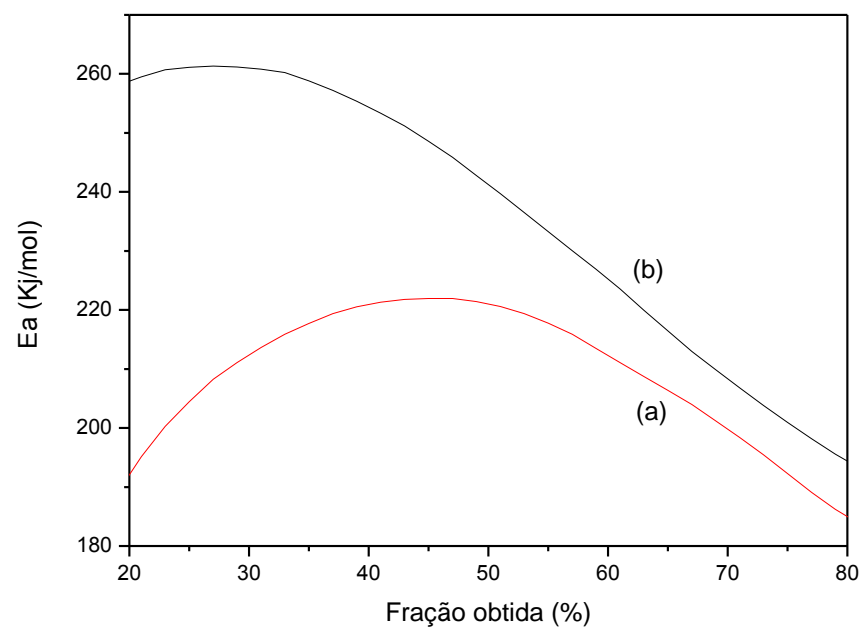

Figura 4 - Energia de Ativação do (a) óleo/Al-MCM-41 e (b) óleo de girassol. 


\section{CONCLUSÕES}

Baseando-se nas técnicas de caracterização empregadas o catalisador mesoporoso, Al-MCM41 com razão $\mathrm{Si} / \mathrm{Al}=50$ sintetizado através do método hidrotérmico com ajuste de $\mathrm{pH}$ diário apresentou a estrutura hexagonal unidimensional típica desses materiais, e a adsorção de nitrogênio mostrou que o material tem área superficial satisfatória para catálise. $\mathrm{O}$ estudo cinético mostrou que a energia de ativação da pirólise do óleo de girassol catalisada pelo material mesoporoso do tipo Al-MCM-41 necessitou de uma menor energia do que a do óleo de girassol sem catalisador, mostrando que a presença do alumínio contribuiu para o aumento de acidez do material.

\section{REFERÊNCIAS}

BECK, J.S.; VARTULI, J.C.; ROTH, W.J.; LEONOWICZ, M.E.; KRESGE, C.T.; SCHMITT, K. D.; CHU, C. T. W.; OLSON, D. H.; SHEPPARD, E. W.; MCCULLEN, S. B.; HIGGINS, Y. B.; SCHELENKER, I. L., A new family of mesoporous molecular-sieves prepared with liquidcrystal templates. Journal American Chemistry Society, v. 114, p.10843, 1992.

DANTAS, H.J.; SOUZA.; A.G.; CONCEIÇÃO, M.M. Estudo Termoanalítico, Cinético e Reológico de Biodiesel Derivado do Óleo de Algodão (Gossypium Hisut).2006. Dissertação (Mestrado em Química) - Centro de Ciências Exatas e da Natureza, Departamento de Química, Programa de Pós-Graduação em Química, Universidade Federal da Paraíba, João Pessoa.

GONZALEZ, W.A.; MACHADO, C.R.; BARRETO, E.J.F.; DALL'OGLIO, E.L., Biodiesel $e$ Óleo Vegetal in Natura Soluções Energéticas para a Amazônia, 2008.

MELO, A. C. R. Craqueamento térmico e termocatalítico do óleo de girassol (Hellianthus annus L.) sobre materiais micro e mesoporosos. 2010. 154f. Tese (Doutorado Química) Departamento de Química, Programa de Pós-Graduação em Química. Universidade Federal do Rio Grande do Norte, Natal.

VYAZOVKIN, S.; GORYACHKO, V. Potentialities of software for kinetic processing of thermoanalytical data by the isoconversion method. Thermochimica Acta, v. 194, p. 221-230, 1992. 